\title{
Parameter Identification of High-Power, High-Voltage Motors Using Electrical Parameter Estimation Function
}

\author{
Yifei Wang ${ }^{1, a}$, Youxin Yuan ${ }^{1, b}$ \\ ${ }^{1}$ School of Automation, Wuhan University of Technology, Wuhan 430070, China \\ aemail: wfynhzs88@163.com, bemail:yyx2000@263.net
}

Keywords: HP HV motors; Parameter Identification; Simulation

\begin{abstract}
An asynchronous motor model in MATLAB/Simulink was selected as the simulation model of high-power, high-voltage (HP HV) motors for its structural suitability. The required electrical parameters of the model (i.e. simulation parameters) were then identified by an appropriate method. Based on the obtained results, a parameter identification system was developed for HP HV motors. The developed system was then applied to a typical HP asynchronous motor YKK1009-4 (12500k, 10kV); results demonstrated the effectiveness of the proposed simulation method.
\end{abstract}

\section{Introduction}

Identification of HP HV motors' parameters is the basis of simulation research on current limited starting of HP HV motors. Using the Asynchronous Machine simulation model of a three-phase asynchronous motor in MATLAB/Simulink (R1012a) requires input of the resistances and self-inductances of the stator and rotor as well as other motor parameters. The technical parameters provided by motor manufacturers include the following items: nominal power, nominal current, speed, efficiency, power factor, etc. Therefore, these required parameters can be obtained only through identification[1][2].

Motor parameter identification can be performed online or offline. Since the starting of a motor is a fast, dynamic process, this study employed an offline identification method. There are two widely-used methods of offline identification: one method involves the no-load and blocked-rotor test, and the other method combines the step response test with the direct current attenuation method[3]-[5]. However, these two methods are inapplicable to high-capacity, high-voltage motors, primarily due to the immense difficulty of conducting such experiments. To address parameter identification of high-capacity, high-voltage motors, this study employed an electrical parameter estimation function in MATLAB/Simulink-PowerSystemTM to obtain the parameters of double squirrel-cage asynchronous motors, including the resistances and self-inductances of stator and rotor, based on the provided technical parameters.

\section{Simulation Model of HP HV Motors and Parameter Identification Method}

Squirrel-cage motors with a power output that exceeds $100 \mathrm{~kW}$ are typically designed with a double squirrel-cage shape. HP HV motors are asynchronous motors with power outputs greater than $500 \mathrm{~kW}$ and a nominal voltage of $6 \mathrm{kV}$ or $10 \mathrm{kV}$; thus, a typical HP HV motor exhibits a double squirrel-cage shape.

The Asynchronous Machine in MATLAB/Simulink (R1012a) was selected as the HP HV motor simulation model; the double squirrel-cage was selected as the rotor type. The primary model parameters include stator resistance Rs, stator leakage inductance L1s, inner cage resistance Rr1, inner cage leakage inductance L1r1, outer cage resistance Rr2, outer cage leakage inductance L1r2, mutual inductance $\mathrm{Lm}$, moment of inertia $\mathrm{J}$, coefficient of viscous friction $\mathrm{F}$, and number of pole pairs P. Figure 1 depicts the steady-state equivalent circuit of a double squirrel-cage asynchronous motor, including the seven electrical parameters described above. 




Fig. 1 Steady-state equivalent circuit of a double squirrel-cage asynchronous motor

Technical parameters provided by manufacturers include the nominal power PN, nominal current $\mathrm{UN}$, rotational speed $\mathrm{SN}$, efficiency $\eta$, power factor $\cos \Phi$, ratio of starting current to nominal current (Ust/UN), ratio of maximum torque to nominal torque (Tst/TN), ratio of blocked-rotor torque to nominal torque ( $\mathrm{Tb} / \mathrm{TN}$ ), and moments of inertia $\mathrm{J}$ of the motor (in both unloaded and loaded states). However, the first seven model parameters must be obtained via parameter identification.

The electrical parameter estimation function for double squirrel-cage asynchronous motors in MATLAB/simulink-PowerSystemTM (i.e., power_Asynchronous MachineParams) can be easily employed to obtain the stator resistance and self-inductance, rotor resistance and self-inductance, and other electrical parameters based on the provided technical parameters.

The employed electrical parameter estimation function for double squirrel-cage asynchronous motors was the Optimization Toolbox solver lsqnonlin. The solution was derived from a non-linear least squares algorithm.

The aforementioned seven electrical parameters were then obtained by solving the non-linear equations described by Eq. (1):

$$
\left\{\begin{array}{l}
f(x)=0 \\
f=\left(f_{1}, f_{2}, f_{3}, f_{4}\right) \\
x=\left(R_{s}, L_{m}, R_{r 1}, L_{1 r 1}, R_{r 2}, L_{1 r 2}\right), L_{1 s}=L_{1 r 2}
\end{array}\right.
$$

where $\mathrm{f}$ can be given by:

$$
\left\{\begin{array}{l}
f_{1}(x)=\frac{T_{n}-T\left(s_{n}\right)}{T_{n}} \\
f_{2}(x)=\frac{T_{n}-T\left(s_{n}\right)}{T_{n}} \\
f_{3}(x)=\frac{p f-p f\left(s_{n}\right)}{p f} \\
f_{4}(x)=\frac{I_{s t}-I\left(s_{n}\right)}{I_{s t}} \\
f_{5}(x)=\frac{T_{b r}-T\left(s_{b r}\right)}{T_{b r}} \\
f_{6}(x)=\frac{T_{s t}-T(1)}{T_{s t}}
\end{array}\right.
$$

In Eq. (2), sn and sbr denote the nominal steady-state error and the blocked-rotor steady-state error, respectively; TN, In, pf, Ist, Ibr, and Tst represent the respective technical parameters of the motor.

The input parameters of the power_Asynchronous MachineParams function include nominal voltage, frequency, nominal current, nominal torque, synchronous speed, nominal speed, ratio of starting current to nominal current, ratio of maximum torque to nominal torque, ratio of blocked rotor torque to nominal torque, and the power factor, all of which represent technical parameters with the exception of nominal torque TN. 
The nominal torque TN can be calculated as follows:

$$
T_{n}=9550 \frac{P_{N}}{S_{N}}
$$

Where PN is the nominal power output $(\mathrm{kW})$ and $\mathrm{SN}$ is the nominal speed (rpm) of the motor.

\section{Parameter Identification System for the Simulation Model of HP HV Motors}

A parameter identification system was developed using MATLAB/Simulink to identify the parameters of the HP HV motor model. The developed system is able to automatically identify parameters and then display and save identification results after the model parameters (i.e. the technical parameters of the motor) have been set. The GUI (graphical user interface) method was then employed to design the primary interface of the parameter identification system for the HP HV motor simulation model. Figures 2 and 3 depict the primary interface and the parameter setting GUI, respectively.

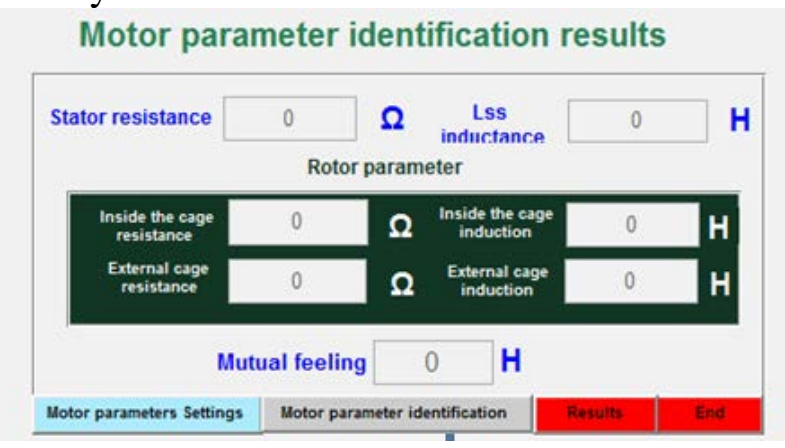

Fig.2. The primary interface

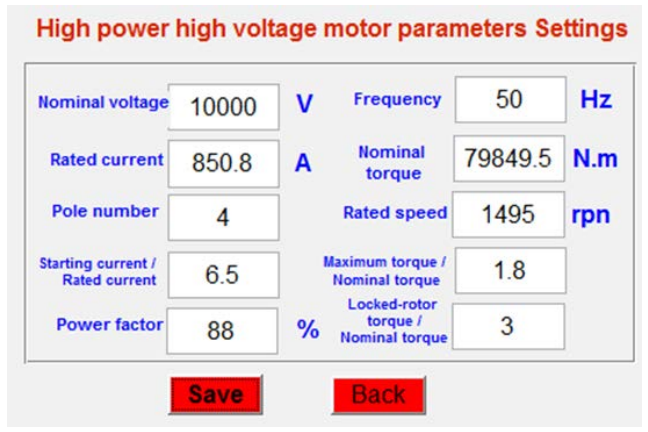

Fig.3. The parameter setting GUI

As shown in Fig. 2, the technical parameters must be set on the technical parameter setting interface after clicking on the "Motor technical parameter setting" button. Then, the motor parameter identification program .m is initiated by clicking on the "Motor parameter identification" button; the results can be saved by clicking on the "Save" button. After the identification is achieved, the operation can be terminated by clicking on the "Finish" button.

As depicted in the GUI presented in Fig. 3, the technical parameters of the motor should first be set and saved by clicking on the "Save" button. The operation can be terminated by clicking on the "Return" button.

The motor parameter identification program .m was used to design the parameter identification programs for the HP HV motor simulation model. The developed programs include the technical parameter loading program and the motor parameter identification program.

$\% * * * * * * * * * * * * * * * * * *$ Load technical parameters

load M_SpecParaSavePara.mat

spec.Vn = M_Para(1) ; nominal voltage/V

spec.fn $\quad$ M_Para $(2) ;$ frequency $/(\mathrm{Hz})$

spec.In = M_Para(3) ; nominal current/A

spec.Tn $\quad$ M_Para(4) ; nominal torque/ N.m

spec.Ns $=60 * 2 *$ spec.fn/M_Para(5) $; \%$ synchronous speed

spec.Nn $\quad$ M_Para(6) ; nominal speed

spec.Ist_In $=$ M_Para(7) ; starting torque to full-load torque ratio

spec.Tst_Tn $=$ M_Para(8) ; starting torque to full-load torque ratio

spec.Tbr_Tn = M_Para(9) 3; breakdown torque to full-load torque ratio

spec.pf $\quad$ M_Para(10) ;pf nominal power factor $/ \%$

$\% * * * * * * * * * * * * * * * * * *$ Motor parameter identification

options.DisplayDetails $=1$;

options.units = 'p.u.';

[params,spec2,errors] = power_AsynchronousMachineParams(spec,options)

options.DrawGraphs = 1; 
options.DisplayDetails = 0;

params = power_AsynchronousMachineParams(spec,options);

\section{Identification of Double Squirrel-cage Asynchronous Motor YKK1009-4 Parameters}

The technical parameters of a double squirrel-cage asynchronous motor YKK1009-4 $(12500 \mathrm{~kW} / 10 \mathrm{kV})$ were input to the newly-developed parameter identification system; results accurately identified the parameters of the motor. Table 1 shows the params outputs, while Table 2 details the spec 2 outputs and errors.

Table 1. Results of YKK1009-4 motor parameter identification (params)

\begin{tabular}{cclc}
\hline No. & Symbol & \multicolumn{1}{c}{ Description } & Output \\
\hline 1 & $R_{s}$ & Stator resistance $/ \Omega$ & 0.0245 \\
2 & $L_{1 s}$ & Stator leakage inductance/H & 0.0337 \\
3 & $R_{r 1}$ & Inner cage resistance $/ \Omega$ & 0.0030 \\
4 & $L_{1 r 1}$ & Inner cage leakage inductance/H & 0.1042 \\
5 & $R_{r 2}$ & Outer cage resistance $/ \Omega$ & 0.2799 \\
6 & $L_{1 r 2}$ & Outer cage leakage inductance/H & 0.0337 \\
7 & $L_{m}$ & Mutual inductance/H & 2.2890
\end{tabular}

Table 2. Results of YKK1009-4 motor parameter identification (spec2 and errors)

\begin{tabular}{cclcc}
\hline No. & Symbol & \multicolumn{1}{c}{ Parameter name } & Output(spec2) & Output(errors/\%) \\
\hline 1 & Vn & Nominal voltage/V & 10000 & \\
2 & Fn & Frequency/(Hz) & 50 & \\
3 & In & Nominal current/A & 850.8 & $4.6648 \times 10^{-4}$ \\
4 & Tn & Nominal torque/N.m & 79850 & $8.9600 \times 10^{-4}$ \\
5 & Vn & Nominal voltage/V & 10000 & \\
6 & Fn & Frequency/Hz & 50 & \\
7 & In & Nominal current/A & 850.8 & $4.6648 \times 10^{-4}$ \\
8 & Tn & Nominal torque/N.m & 79850 & $8.9600 \times 10^{-4}$ \\
9 & Ns & Synchronous speed & 1500 & \\
10 & Nn & Nominal speed/rpm & 1495 & \\
11 & Ist_In & Starting current to nominal current ratio & 6.5 & 0.0059 \\
12 & Tst_Tn & Starting torque to nominal torque ratio & 1.8 & -0.0019 \\
13 & Tbr_Tn & Blocked-rotor torque to nominal torque ratio & 3 & -0.0018 \\
14 & Pf & Power factor/\% & 88 & $-6.8034 \times 10^{-5}$ \\
15 & P & Number of pole pairs & 2 & \\
16 & We & Nominal angular speed/rsd/s & 314.1593 & \\
17 & Vin & Nominal phase voltage & 5775 & \\
18 & Sn & Nominal steady-state error & 0.0033 & \\
19 & Ist & Starting current & 5530.2 & 0.0063 \\
20 & Tst & Starting torque & 143730 & -0.0010 \\
21 & Tbr & Breakdown torque & 239550 & $-8.8604 \times 10^{-4}$ \\
22 & Pn & Nominal power/W & 1250100 & \\
23 & Ns & Synchronous speed/rpm & 1500 & \\
\hline
\end{tabular}

*The non-zero error values in Table 2 represent errors in other technical parameters of the motor obtained by identification. 


\section{Conclusion}

This study employed the Asynchronous Machine simulation model of a three-phase asynchronous motor in MATLAB Simulink as an HP HV motor simulation model in order to determine the electrical parameters of a double squirrel-cage motor. The double squirrel-cage was selected as the rotor type to suit the motor's structure.

After developing an identification method, the electrical parameter estimation function in MATLAB/simulink-PowerSystemTM for double squirrel-cage asynchronous motors (i.e., power_Asynchronous MachineParams) was used to obtain the stator resistance and self-inductance, rotor resistance and self-inductance, and other parameters of double squirrel-cage asynchronous motors based on the technical parameters provided by the motor manufacturer.

An HP HV motor parameter identification system was developed using GUI. The developed system is able to automatically and accurately identify the simulation model parameters after the technical parameters provided by motor manufacturers are input.

The proposed parameter identification system was then applied to the parameter identification of a typical HP asynchronous motor YKK1009-4 (12500k, 10kV). The results were satisfactory, with a maximum error of $0.0063 \%$, demonstrating the effectiveness of the simulation method proposed in this study. Therefore, the three-phase asynchronous motor simulation model and the verified results can lay a foundation for research on the current limited starting of HP HV motors.

\section{Acknowledgement}

In this paper, the research was sponsored by the projects of Hubei Science and Technology Plans of China (Granted No: 2014BAA011 and 2013BAA009).

\section{References}

[1] [Gang Zhao, Lei Wang. Research and simulation base on asynchronous motor soft starter [J]. Advanced Materials Research, 2012: 3615 - 3619

[2] Yiping Xiao, Youxin Yuan. An Integrated System of Wound Rator Induction Motor Soft Starting and Doubly-fed Speed Governing. 2010 International Conference on Electrical and Control Engineering[C]. Wuhan: IEEE Computer Society, 2010: 1550 - 1553

[3] Zhang Hu, Li Zhengxi, Tong Chaonan. Off-line Parameter identification of induction motor based on recursive least-squares algorithm [J]. Chin. Soc. for Elec. Eng., 201131 (18) 79-86.

[4] Yanhui He, Yue Wang. Parameter identification of an induction machine at standstill using the vector constructing method [J]. IEEE Transactions on Power Electrons, 2012 27(2) 905-915.

[5] Christiaan Moons, Bart De Moors. Parameter identification of induction motor drives [J]. Aummalm, 1995 31(8) 1137-1147. 